\title{
Continuous Markov Random Field Optimization using Fusion Move Driven Markov Chain Monte Carlo Technique
}

\author{
Wonsik Kim and Kyoung Mu Lee \\ Department of EECS, ASRI, Seoul National University, 151-742, Seoul, Korea \\ Homepage: http://cv.snu.ac.kr \\ Email: $\{$ ultra16, kyoungmu\}@snu.ac.kr
}

\begin{abstract}
Many vision applications have been formulated as Markov Random Field (MRF) problems. Although many of them are discrete labeling problems, continuous formulation often achieves great improvement on the qualities of the solutions in some applications such as stereo matching and optical flow. In continuous formulation, however, it is much more difficult to optimize the target functions. In this paper, we propose a new method called fusion move driven Markov Chain Monte Carlo method (MCMC-F) that combines the Markov Chain Monte Carlo method and the fusion move to solve continuous MRF problems effectively. This algorithm exploits powerful fusion move while it fully explore the whole solution space. We evaluate it using the stereo matching problem. We empirically demonstrate that the proposed algorithm is more stable and always finds lower energy states than the state-of-the art optimization techniques.
\end{abstract}

Keywords-Markov Random Field; Markov Chain Monte Carlo; Stereo Matching;

\section{INTRODUCTION}

MRF model have achieved great success in many vision applications [1]. Although most of them have been formulated as discrete labeling problems, continuous formulation of the problem often achieves great improvement on the qualities of the solutions in some applications such as stereo matching and optical flow.

However, continuous formulation make it much more difficult to optimize the target function compared to the fact that we have many powerful discrete optimizer such as Graph Cuts [2], [3], [4] and Message Passing methods [5], [6]. There are two dominating approaches to solve the continuous optimization problems. First approach is to model the problems as convex [7] which are easy to optimize. Despite of its success, it is limited by the fact that it cannot allow non-convex energy models. Second approach is to apply powerful discrete optimization algorithms to some reduced discrete solution spaces [8], [9]. This approach is also limited by the fact that it cannot fully explore the original solution space. So it only provides approximated solutions and cannot sufficiently lower the energy of the solutions.

In this paper, we propose a powerful optimization technique that directly solves the continuous MRF problems. It combines two powerful methods: Markov Chain Monte
Carlo method (MCMC) and Quadratic Pseudo-Boolean Optimization (QPBO) fusion move. The idea to combine stochastic methods and deterministic methods was proposed by Kim and Lee [10]. They, however, applied their method only to the discrete MRF optimization. To deal with continuous MRF optimization, we propose a new method, called Fusion Move driven Markov Chain Monte Carlo (MCMCF). It exploits powerful deterministic methods in the framework of sampling-based stochastic method. The samples can rapidly move to lower energy state owing to deterministic methods. Also, it can effectively jump from one basin to another over the energy barrier. Consequently, this property increases mixing rate and yields faster convergence and better solutions. Moreover, exploration is not restricted in the reduced space. To demonstrate the effectiveness of the algorithm, MCMC-F is applied to stereo matching problems.

\section{ENERGY FORMULATION}

To evaluate the performance of MCMC-F on stereo problem, we design a posterior probability of the disparity map that is composed of two terms: data term and smoothness term. The formulation is as follows.

$$
\begin{aligned}
p\left(\mathbf{x} \mid I_{0}, I_{1}\right)= & \frac{1}{Z} \prod_{p \in \mathcal{V}} \exp \left\{-d_{p}\left(x_{p}\right)\right\} . \\
& \prod_{(p, q) \in \mathcal{E}} \exp \left\{-V_{p q}\left(x_{p}, x_{q}\right)\right\},
\end{aligned}
$$

where $\mathbf{x}=\left\{x_{1}, x_{2}, \cdots, x_{N}\right\}$ is the disparity map, $x_{p}$ denotes the disparity of the pixel $p, I_{0}$ and $I_{1}$ is left and right images, and the set $\mathcal{V}$ and $\mathcal{E}$ contain the nodes and the edges in the MRF model, respectively.

The data term $d_{p}$ is defined as follows.

$$
d_{p}\left(x_{p}\right)=\left\|I_{0}(p)-I_{1}\left(p+x_{p}\right)\right\|^{2},
$$

where $\|\cdot\|$ is the Euclidean distance of the RGB color values. Since the disparity $x_{p}$ can have real value, we linearly interpolate the pixel values to compute $I_{1}\left(p+x_{p}\right)$.

For the smoothness term $V_{p q}$, we assume 4-neighborhood system, and we define 


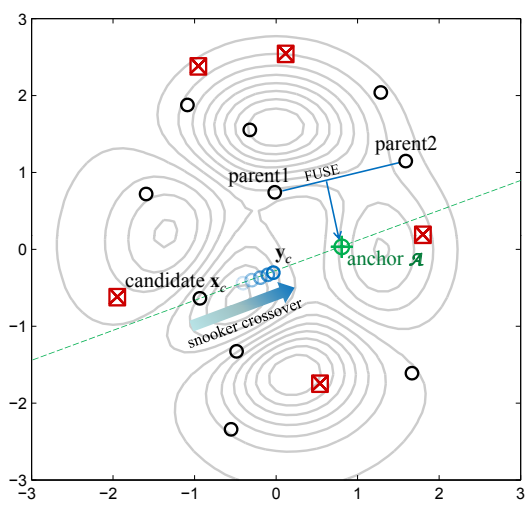

(a)

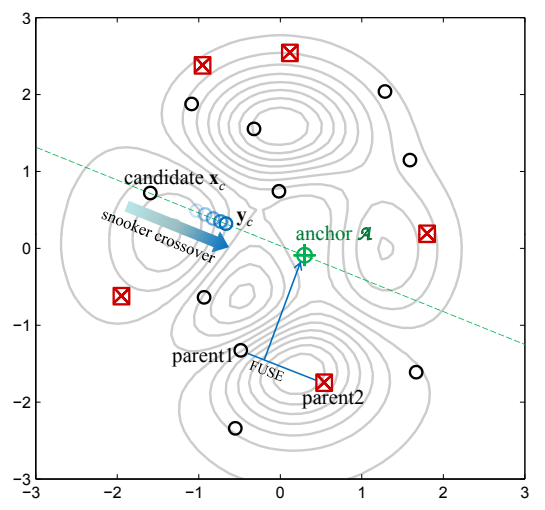

(b)

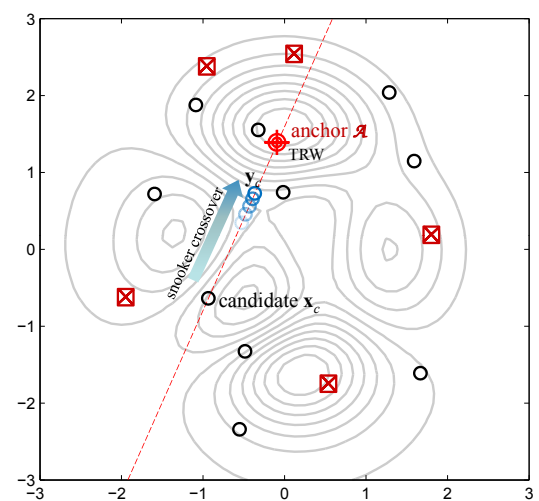

(c)

Figure 1. Three types of anchor generations and snooker crossover: (a) dynamic anchor with both parents from the samples, (b) dynamic anchor with one parent from the samples and the other from the proposals, and (c) static anchor.

$$
V_{p q}\left(x_{p}, x_{q}\right)=\lambda_{p q} \log \left(1+\frac{1}{2 \nu^{2}}\left|x_{p}-x_{q}\right|^{2}\right) .
$$

We design the smoothness penalty to be the negative $\log$ of a Student-t function. The parameter $\nu$ controls the degree of freedom. The weight $\lambda_{p q}$ varies according to the color difference between neighboring pixels so that we enforce disparity discontinuities to coincide with image color discontinuities.

\section{ENERGy Minimization}

In this section, we describe how to optimize aforementioned energy function. We briefly review the samplingbased optimization, and then we explain the detail of our MCMC-F algorithm.

\section{A. Sampling-based Optimization}

Sampling-based optimization exploits the sampling method to obtain optimum solution of the energy function. MCMC methods have been used to sample from a given distribution $p(\mathbf{x})$. For the optimization problem, MCMC is often embedded into the Simulated Annealing framework. However, since most MCMC methods allow only local moves, in a large solution space it takes a very long time to reach the global optimum.

To overcome the limitations of MCMC, Population-Based MCMC (Pop-MCMC) has been applied to the vision problem [11]. In Pop-MCMC, multiple samples are drawn from

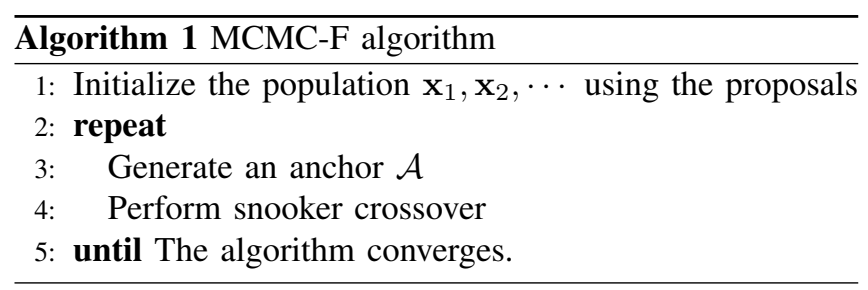

multiple Markov chains. To obtain the optimum sample, Parallel Tempering is used instead of Simulated Annealing. It generates multiple chains in parallel according to several different temperatures, and exchanges information among them to accelerate the mixing rate. The target distribution of $i$ th chain is defined as follows.

$$
p_{i}\left(\mathbf{x}_{i}\right)=p\left(\mathbf{x}_{i}\right)^{\frac{1}{T_{i}}},
$$

where $\mathbf{x}_{i}$ is the sample of the $i$ th chain, $p(\mathbf{x})$ is an original target distribution, and $T_{i}$ is the temperature of the $i$ th chain.

\section{B. MCMC combined with Fusion move}

The main idea of MCMC-F is to combine MCMC method and the QPBO fusion move. To combine them, we follow the strategy which has been introduced by Kim and Lee [10]. Using this strategy, we have designed the algorithm to optimize the energy function formulated in section II.

As in Pop-MCMC, we derive multiple samples from multiple chains. Each sample represents a single disparity map. Those samples are iteratively updated to obtain the optimum solution in the main body of the MCMC-F algorithm. The main body is composed of two phases: anchor generation and snooker crossover. Overall algorithm of MCMC-F is summarized in Algorithm 1.

Initialization We initialize samples using plane fitting method for stereo matching. We firstly obtain 14 segmentation results of the reference (left) image using different methods [12], [13] and different parameters. And then we calculate initial disparities for each pixel using the Sum of Absolute Difference (SAD) and Winner Takes All (WTA) schemes. Incorrectly estimated disparities are eliminated by the disparity crosschecking method [14]. After that, for each segment we estimate a plane by the least squares method with reliable disparity values. After we estimated the plane, we further identify more outlier 

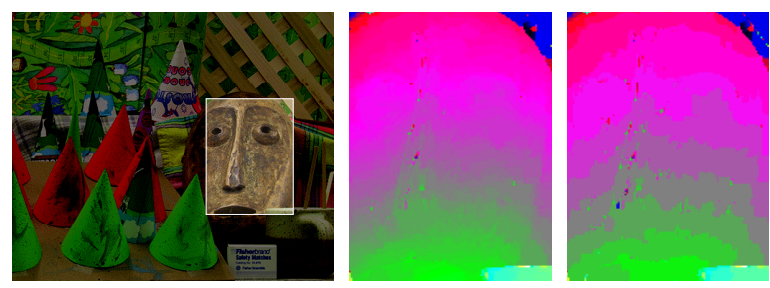

(a) Cones
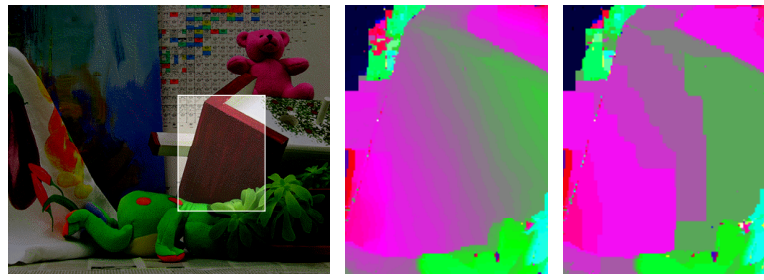

(b) Teddy

Figure 2. Close view of the resulting disparity maps. First column is reference image, second column is results of MCMC-F, and third column is results of TRW. (Best viewed in color.)

disparities that do not fit to the plane. We iteratively re-estimate the exact plane only using remaining inliers. We finally obtain 14 different disparity maps which are called proposals henceforth. Each sample in MCMC-F is initialized to one of the proposal disparity maps at random.

Anchor generation In the MCMC-F algorithm, there are three different types of anchors. The first two are the dynamic anchors. When generating dynamic anchors, we first select two samples as parents. And then, we generate a new disparity map through QPBO fusion of the parents. The solution of QPBO is the dynamic anchor. This anchor is dynamically generated and destroyed while the algorithm is running. Instead of selecting two parents from samples, we can select one of the parent from samples and another from proposals. The third type of the anchor is the static anchor. To generate the static anchors, we initially run non-move-making deterministic algorithms such as the Tree ReWeighted Message Passing (TRW) and Belief Propagation (BP) before MCMC-F starts. Those solutions are used as the anchor points while the algorithm is running. Three types of anchor generation are illustrated in Fig. 1.

For each iteration, we generate a dynamic anchor with the probability $Q_{D M}$, and a static anchor with the probability $1-Q_{D M}$.

Snooker crossover After an anchor is chosen, we update the samples by using snooker crossover. For every samples (except parent) as a candidate $\mathbf{x}_{c}$, we perform snooker crossover with the anchor point $\mathcal{A}$. Newly generated sample $\mathbf{y}_{c}$ lies on the line going through the candidate and

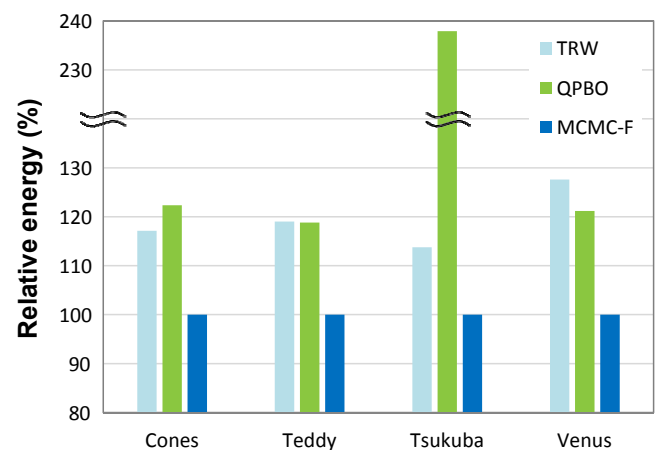

Figure 3. Final energy obtained by each algorithm.

the anchor according to:

$$
\mathbf{y}_{c}=\kappa s^{r} \mathbf{x}_{c}+\left(1-\kappa s^{r}\right) \mathcal{A},
$$

where $\kappa, r$ and $s$ are random variables which control the dynamics of snooker crossover. They are designed to satisfy the reversibility condition of Markov chain. In our implementation, the parameters $\kappa$ and $r$ are randomly chosen among +1 and -1 with equal probability. With the probability of 0.5 , the parameter $s$ has the value $s=s_{\min }$ and with the probability of 0.5 , the parameter $s$ is uniformly drawn from $\left(s_{\min }, s_{\max }\right]$.

After the new sample $\mathbf{y}_{c}$ is generated, the candidate $\mathbf{x}_{c}$ is substituted with $\mathbf{y}_{c}$ according to the acceptance probability:

$$
\alpha=\min \left(1, \frac{p_{c}\left(\mathbf{y}_{c} \mid I_{0}, I_{1}\right)}{p_{c}\left(\mathbf{x}_{c} \mid I_{0}, I_{1}\right)}\right) .
$$

where $p_{c}$ is the target distribution of $c$ th chain.

\section{EXPERIMENTS}

To demonstrate the performance of the proposed method, we experimented on 4 Middlebury test images: Cones, Teddy, Tsukuba, and Venus [15]. We compared the performance of MCMC-F, QPBO, and TRW [5], [6]. For TRW, the original problems were reduced so that it had only integer values as labels since we cannot directly apply TRW to the continuous optimization problems. The solution of TRW was used for the static anchor. The running time of MCMC-F, and TRW was set to 1,000 seconds. QPBO took $75 \sim 90$ seconds to terminate. All the experiments were performed on the Intel Quad Core 2.4GHz PC platform. In all experiments, the parameters was fixed as follows: for MCMC, population size was $100, Q_{D M}$ was set to 0.9 , the temperature of $i$ th chain was set to $i \times 100$; for the energy function, $\nu=0.2$ and $\lambda_{p q}=150$ if the sum of absolute differences between $I_{0}(p)$ and $I_{0}(q)$ was less than or equal to 30 , and $\lambda_{p q}=50$ otherwise. 


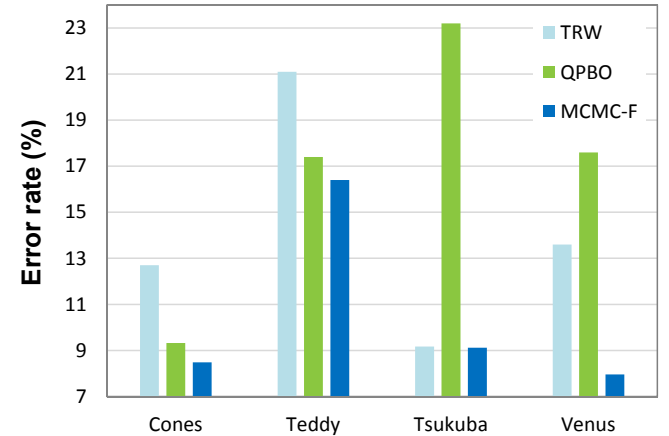

Figure 4. Error rate on the un-occluded region of each disparity map with error threshold of 0.5 .

Some of the resulting disparity maps are shown in Fig. 2. Final energies and the error rates of the disparity maps are depicted in Fig. 3 and Fig. 4, respectively. Relative energies were computed by $100 \times$ (energy of solution)/(minimum energy). We note that the results of TRW have severe artifacts due to quantization. Because the solution of TRW can have only integer values as labels, disparities of the slanted objects are presented as piece-wise fronto parallel planes. TRW cannot fully explore the continuous solution spaces. On the contrary, MCMC-F produces much more natural disparity maps. It is also shown that MCMC-F always finds the lowest energy solutions which have minimum error rates.

The properties of the solution spaces differ from image to image. For example, TRW solutions have lower energy values than QPBO solutions for Cones and Tsukuba images. On contrary, TRW solutions have higher energy values for Teddy and Venus images. Despite of that, MCMC-F achieves the lowest energy among all the algorithms for every test image.

\section{CONCLUSION}

MRF optimization has achieved great success over wide range of applications. In some applications such as stereo matching and optical flow, continuous formulation enables to obtain much more realistic results. However, so far, existing continuous MRF optimization methods have limitations in application to real problems. In this paper, we proposed MCMC-F algorithm to effectively optimize functions in continuous spaces. It is sampling-based optimization algorithm that is combined with fusion move. It exploits powerful discrete optimization methods while it has ability to fully explore the continuous solution space. Since samples can move to lower energy state where it is impossible to be reached by the conventional approaches, it can find lower energy state than other algorithms. We experimentally demonstrate that it achieves significantly lower energy state solutions than QPBO and TRW. Furthermore, since MCMC$\mathrm{F}$ can be applied to any type of general energy functions, it will be useful to solve many other applications. And this work will also help to design more realistic complex energy models.

\section{REFERENCES}

[1] R. Szeliski, R. Zabih, D. Scharstein, O. Veksler, V. Kolmogorov, A. Agarwala, M. Tappen, and C. Rother, "A comparative study of energy minimization methods for markov random fields with smoothness-based priors," PAMI, vol. 30, no. 6, pp. 1068-1080, 2008.

[2] Y. Boykov, O. Veksler, and R. Zabih, "Fast approximate energy minimization via graph cuts," PAMI, vol. 23 , no. 11 , pp. 1222-1239, 2001.

[3] V. Kolmogorov and R. Zabih, "What energy functions can be minimized via graph cuts?" PAMI, vol. 26, no. 2, pp. 147159, 2004.

[4] Y. Boykov and V. Kolmogorov, "An experimental comparison of min-cut/max-flow algorithms for energy minimization in vision," PAMI, vol. 26, no. 9, pp. 1124-1137, 2004.

[5] M. Wainwright, T. Jaakkola, and A. Willsky, "Map estimation via agreement on (hyper)trees: Message-passing and linearprogramming approaches," IEEE Trans. Information Theory, vol. 51, no. 11, pp. 3697-3717, 2005.

[6] V. Kolmogorov, "Convergent tree-reweighted message passing for energy minimization," $P A M I$, vol. 28 , no. 10, pp. 1568-1583, 2006.

[7] N. Papenberg, A. Bruhn, T. Brox, S. Didas, and J. Weickert, "Highly accurate optic flow computation with theoretically justified warping," PAMI, vol. 67, no. 2, pp. 141-158, 2006.

[8] O. J. Woodford, P. H. S. Torr, I. D. Reid, and A. W. Fitzgibbon, "Global stereo reconstruction under second order smoothness priors," in CVPR, 2008.

[9] V. Lempitsky, S. Roth, and C. Rother, "Fusionflow: Discretecontinuous optimization for optical flow estimation," in CVPR, 2008.

[10] W. Kim and K. M. Lee, "Markov chain monte carlo combined with deterministic methods for markov random field optimization," in CVPR, 2009.

[11] W. Kim, J. Park, and K. Lee, "Stereo matching using population-based mcmc," IJCV, vol. 30, no. 6, pp. 1068-1080, 2008.

[12] D. Comaniciu and P. Meer, "Mean shift: A robust approach toward feature space analysis," PAMI, vol. 24, no. 5, pp. 603619, 2002.

[13] P. F. Felzenszwalb and D. P. Huttenlocher, "Efficient graphbased image segmentation," IJCV, vol. 59, no. 2, pp. 167-181, 2004.

[14] L. Hong and G. Chen, "Segment-based stereo matching using graph cuts," in $C V P R, 2004$, pp. 74-81.

[15] “http://vision.middlebury.edu/stereo/." 